Research Article

\title{
Treatment of Glucocorticoid-Induced Osteoporosis and Risk Factors for New Vertebral Fractures in Female Patients with Autoimmune Diseases
}

\author{
Koichiro Shinoda $(D)$ and Hirofumi Taki \\ First Department of Internal Medicine, University of Toyama, 2630 Sugitani, Toyama 930-0194, Japan \\ Correspondence should be addressed to Koichiro Shinoda; koichiro@med.u-toyama.ac.jp
}

Received 12 March 2021; Accepted 16 October 2021; Published 25 October 2021

Academic Editor: Rimesh Pal

Copyright (C) 2021 Koichiro Shinoda and Hirofumi Taki. This is an open access article distributed under the Creative Commons Attribution License, which permits unrestricted use, distribution, and reproduction in any medium, provided the original work is properly cited.

\begin{abstract}
We aimed to evaluate the compliance of physicians with the 2014 guidelines of the Japanese Society for Bone and Mineral Research, for the prevention and treatment of glucocorticoid (GC) induced osteoporosis (GIO) and to investigate the risk of fracture and other associated risk factors in bisphosphonate-treated patients. We evaluated 90 female patients with nonrheumatoid arthritis autoimmune diseases who received long-term GC treatment ( $\geq 12$ months). Clinical characteristics, including age, GC dose, history of fragility fractures, osteoporosis treatments, as well as lumbar (L2-L4) and femoral neck bone mineral density, were collected from the patients' medical charts. New vertebral fractures during the study period were evaluated using thoracic and lumbar spine radiographs by quantitative measurements. The GIO score was calculated for each patient according to 2014 Japanese guidelines. Of the 90 patients evaluated, 60 were indicated for osteoporosis treatment, based on the 2014 guidelines of Japan. We observed a high compliance rate, with $93 \%$ of patients receiving osteoporosis treatment and $50 \%$ receiving bisphosphonates. In total, eight patients developed new vertebral fractures during the study, six of whom received bisphosphonates. In bisphosphonate-treated patients, fracture risk was associated with GC treatment and a lack of active vitamin D3 supplementation. The compliance rate with the updated Japanese 2014 guidelines at our institution was very high. Large randomized controlled trials are needed to confirm our findings that suggest that active vitamin D3 should be used in combination with bisphosphonates for the treatment of GIO to reduce fracture risk.
\end{abstract}

\section{Introduction}

Glucocorticoids (GCs), which have anti-inflammatory and immunosuppressive effects, are widely used to treat various diseases, including autoimmune disorders. However, long-term GC therapy may have various adverse effects. GC-induced osteoporosis (GIO) is the most common of these, and $30 \%-50 \%$ of patients who receive long-term GC therapy develop fractures $[1,2]$. GIO is characterized by a dose-dependent risk of fracture and loss of bone mineral density (BMD) in the lumbar spine and femoral neck that peaks during the first 3-6 months of GC treatment [2]. Fracture risk is higher in patients with GIO than in those with postmenopausal osteoporosis at similar baseline BMD levels, and fractures can occur with minimal or no loss of BMD $[3,4]$. Therefore, early preventive measures against GIO are essential in patients under long-term GC therapy.

In 2004, the Japanese Society for Bone and Mineral Research (JSBMR) released clinical guidelines for GIO treatment in patients aged 18 years or older, who planned to use oral GCs for over 3 months [5]. The JSBMR 2004 guidelines recommend antiosteoporosis treatment for patients with previous, or new fragility fractures, as well as those with a $\mathrm{BMD} \leq 80 \%$ of the young adult mean (YAM) [5]. However, adherence to these guidelines was only $23.3 \%$, possibly because of the low rate of BMD measurements in clinical settings [6]. In 2014, the JSBMR identified age, GC dose, lumbar spine BMD, and a history of fragility fractures as independent risk factors for new fractures and 
incorporated these risk factors into a new scoring system to increase compliance [7].

Based on the JSBMR 2014 guidelines, fracture risk is calculated as the sum of the scores for each risk factor, and pharmacological prevention and treatment for GIO are recommended in patients who reach a total score of 3 or more (Figure 1) [7]. Furthermore, patients aged 65 years or older, with prior fragility fractures, receiving a GC dose of $7.5 \mathrm{mg} /$ day or higher, or those with a lumbar spine BMD $<70 \%$ of YAM, are considered to be at risk for future fractures and should therefore receive treatment for osteoporosis [7]. Treatment with bisphosphonates, such as alendronate, risedronate, and zoledronic acid, with concomitant vitamin D and calcium supplementation, is recommended as the first-line treatment option for GIO [8]. Although all three treatments inhibited lumbar and hip BMD loss, only alendronate and risedronate reduced the rate of vertebral fractures [9-16]. However, people taking bisphosphonates may be more susceptible to osteonecrosis of the jaw (ONJ) [17] and atypical femoral fractures [18, 19]. In addition, although bisphosphonates have not been known to impact pregnancy or cause fetal malformation $[20,21]$, there is still insufficient evidence for the same, and the American College of Rheumatology does not recommend bisphosphonate treatment for those planning to become pregnant [22]. Alternative treatments for GIO include denosumab $[23,24]$, teriparatide $[25,26]$, and active vitamin D3 monotherapy [27]. However, whether clinicians adhere to the JSBMR 2014 guidelines and recommend osteoporosis treatment accordingly remains unknown.

In this observational study, we investigated compliance with the JSBMR 2014 guidelines in patients with autoimmune diseases other than rheumatoid arthritis (RA). In addition, we identified six bisphosphonate-treated patients who developed new vertebral fractures during the study period and determined the fracture risk factors for these patients.

\section{Materials and Methods}

2.1. Participants. In this single-center, retrospective study, we examined all female patients with autoimmune diseases other than RA, who visited the author's specialized outpatient clinic at the Department of Rheumatology and Clinical Immunology at Toyama University Hospital, from April to December 2017. A total of 97 patients underwent long-term GC treatment ( $\geq 12$ months). We included patients who had at least two thoracic and lumbar spine radiographs (frontal and lateral views) and had $\mathrm{BMD}$ measurements made by dualenergy X-ray absorptiometry of the lumbar spine and femoral neck, at the initial and final examinations at our hospital. There were no other exclusion criteria. Study approval was obtained from the Ethics Committee of Toyama University Hospital (no. R2017161). The patients provided informed consent via an opt-out form provided on our hospital website.

2.2. Data Collection. Data were collected from the patients' medical charts, from the start of GC therapy to December 31, 2017. The data collected included age, underlying disease, disease duration, maximum and current daily dose of GC, osteoporosis treatment and duration, prior fragility fractures, lumbar L2-4 BMD (\%YAM), and femoral neck BMD (\%YAM). Adverse events due to osteoporosis medication, including $\mathrm{ONJ}$ and atypical femoral fractures, were also observed.

New vertebral fractures during the study period were evaluated using thoracic and lumbar spine radiographs (frontal and lateral views), by manual quantitative measurements. The diagnosis of vertebral fractures was based on the criteria for vertebral fractures (1996 version) [28]. For patients who developed new vertebral fractures during the study period, data were collected at the time of fracture, except for BMD, for which data were collected at the final measurement, before the fracture. Data were collected from the time of the final BMD measurement for patients who did not have a new vertebral fracture.

2.3. GIO Scoring. For each patient, the GIO score was calculated based on the JSBMR 2014 guidelines [7], using lumbar or femoral neck BMD measurements. New fractures during the study period were not included in the GIO scores.

2.4. Statistical Analysis. The clinical characteristics of bisphosphonate-treated patients, with or without fractures were compared using the $t$-test or $\chi^{2}$-test. Logistic regression analysis was performed using new fractures as the dependent variable and each risk factor as an independent variable. $\mathrm{JMP}^{\circledR}$ software (version 13, SAS Institute Inc.) was used for all statistical analyses, and $p$ values $<0.05$ were considered as statistically significant.

\section{Results}

3.1. Subject Characteristics. Of the 152 female patients who visited our clinic for autoimmune diseases other than RA between April and December 2017, 90 met the inclusion criteria and were included in our study. The clinical characteristics of the 90 female patients receiving long-term GC treatment ( $\geq 12$ months) are shown in Table 1 . The final GC dose was $<5 \mathrm{mg} /$ day in $30 \%, 5-7.5 \mathrm{mg} /$ day in $40 \%$, and $\geq 7.5 \mathrm{mg} /$ day in $30 \%$ of the patients. Eleven patients had a history of vertebral fracture before the initial examination at our hospital. The mean lumbar and femoral neck BMD measurements recorded at the final observation were $95.7 \%$ and $87 \%$, respectively.

3.2. JSBMR 2014 Guideline Adherence. To determine the guideline adherence, we first calculated the GIO scores for GC-treated patients using the JSBMR 2014 guidelines (Figure 1) [7]. According to these guidelines, 57 (63\%) and $60(66 \%)$ patients had a GIO score of 3 or higher when scores were based on lumbar or femoral neck BMD measurements, respectively, and were therefore indicated for osteoporosis treatments (Table 2).

Of the 60 patients with a GIO score of 3 or higher, 56 (93\%) received osteoporosis treatment (Table 2). Thirty 


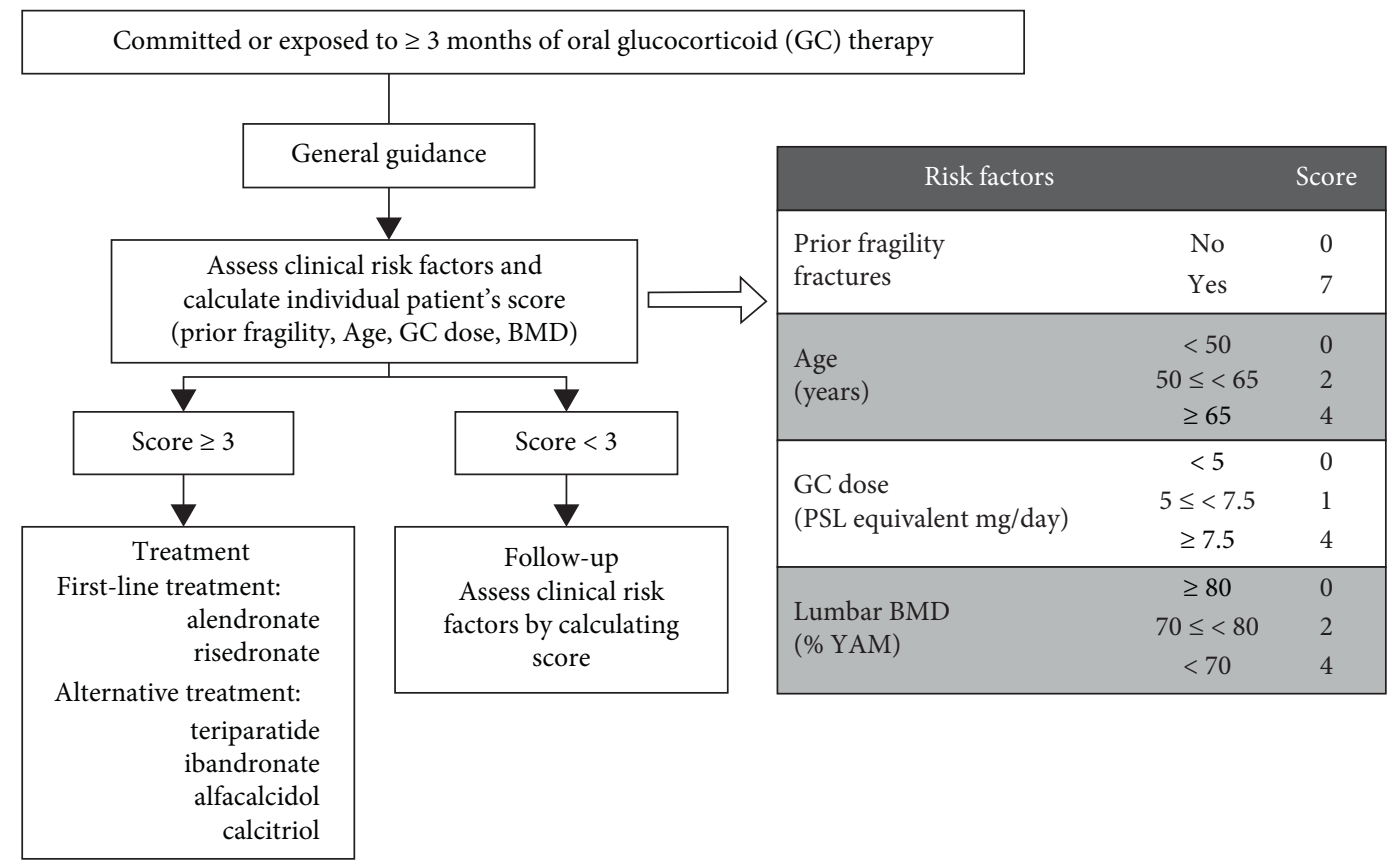

FIgURE 1: Osteoporosis treatment for patients identified as high-risk for glucocorticoid-induced osteoporosis.

TABle 1: Participant characteristics.

\begin{tabular}{lc}
\hline & Total $(n=90)$ \\
\hline Age (years), mean (SD) & $52.9 \pm 14.5$ \\
Sex, female (\%) & $90(100)$ \\
\hline Underlying diseases, no. (\%) & \\
SLE & $36(40.0)$ \\
Inflammatory myopathy & $14(15.5)$ \\
Scleroderma & $9(10)$ \\
MCTD & $8(8.9)$ \\
Sjogren's syndrome & $5(5.6)$ \\
Vasculitis & $6(6.7)$ \\
Others & $12(13.3)$ \\
\hline Current glucocorticoid dose, no. (\%) & \\
$<5$ mg/day & $27(30)$ \\
$5 \leq x<7.5$ mg/day & $36(40)$ \\
$\geq 7.5$ mg/day & $27(30)$ \\
Prior fragility fracture (s), no. (\%) & $11(12.2)$ \\
\hline BMD (\% YAM), mean (SD) & \\
Lumbar spine & $95.7(12.7)$ \\
Femoral neck & $87(12)$ \\
\hline Glucocorticoid-induced osteoporosis score, lumbar $\mathrm{BMD}$ \\
$<3$, no. (\%) & $33(37)$ \\
$3-5$, no. (\%) & $25(28)$ \\
$\geq 6$, no. (\%) & $32(36)$ \\
\hline Glucocorticoid-induced osteoporosis score, femoral neck BMD \\
$<3$, no. (\%) & $30(34)$ \\
$3-5$, no. (\%) & $23(26)$ \\
$\geq 6$, no. (\%) & $37(40)$ \\
\hline
\end{tabular}

SLE, systemic lupus erythematosus; MCTD, mixed connective tissue disease; YAM, young adult mean. Glucocorticoid-induced osteoporosis scores were determined as described previously [7].

patients received bisphosphonate treatment, with 15 of these patients receiving concomitant active vitamin D3 (alfacalcidol or eldecalcitol), the only approved vitamin D3
TABLE 2: Osteoporosis treatment for patients identified as high-risk for glucocorticoid-induced osteoporosis.

\begin{tabular}{lc}
\hline & Prescribed no. (\%) \\
\hline Osteoporosis treatment & $56(93)$ \\
Bisphosphonates & $30(50)$ \\
With active vitamin $\mathrm{D}_{3}$ & $15(25)$ \\
Without active vitamin $\mathrm{D}_{3}$ & $15(25)$ \\
Teriparatide & $3(5)$ \\
Denosumab with vitamin $\mathrm{D}_{3}$ & $12(20)$ \\
Active vitamin $\mathrm{D}_{3}$ monotherapy & $11(18)$ \\
Alfacalcidol & $7(12)$ \\
Eldecalcitol & $4(6)$ \\
Untreated & $4(6)$ \\
\hline
\end{tabular}

supplement in Japan. In addition, 12 patients received denosumab, 3 received daily subcutaneous injections of teriparatide, and 11 received active vitamin D3 (alfacalcidol or eldecalcitol) monotherapy. Only four patients (6\%) did not receive any osteoporosis treatment.

Next, we investigated the reasons behind why some patients did not receive osteoporosis treatment and why some received only active vitamin D3. In the untreated patients, two refused treatment, whereas in the other two cases, the physician did not prescribe osteoporosis medication, for which no reason was provided. Of the 11 patients who received only active vitamin D3, two were planning to become pregnant, three were receiving dental treatments, two had experienced side effects when using bisphosphonates, and the remaining four were not prescribed bisphosphonates by the attending physician.

Adverse events for patients who received osteoporosis medication included two patients with ONJ (one receiving bisphosphonates and one receiving denosumab) and one patient with an atypical femoral fracture (receiving 
bisphosphonates). The patients with ONJ had been on glucocorticoid therapy for 25 and 19 years and were treated with $15 \mathrm{mg}$ or $12.5 \mathrm{mg} /$ day of prednisolone, respectively, when they developed ONJ. The patient with an atypical femoral fracture had been on glucocorticoid therapy for 11 years and was treated with $5 \mathrm{mg} /$ day of prednisolone, after which she developed an atypical femoral fracture.

3.3. Development of New Vertebral Fractures. An additional eight patients developed vertebral fractures during the study period (Table 3), and no other fractures were detected in this patient population. Of these, two patients received a high dose of GC $(\geq 10 \mathrm{mg} /$ day), but had not been treated with bisphosphonates. A 34-year-old patient avoided bisphosphonate treatment because of pregnancy concerns, whereas a 49 -year-old patient did not receive bisphosphonate treatment at the physician's discretion. The remaining six patients developed new vertebral fractures after bisphosphonate treatment. Vertebral fractures occurred in high-risk patients with a high GIO score of $\geq 4$ points.

3.4. Vertebral Fracture Risk Factors in BisphosphonateTreated Patients. To determine the vertebral fracture risk factors in bisphosphonate-treated patients, we compared the clinical characteristics of the six bisphosphonate-treated patients with new vertebral fractures with the 24 bisphosphonate-treated patients without new vertebral fractures (Table 4). There was a trend towards an increase in age and disease duration in patients with vertebral fractures compared to those without vertebral fractures; however, this difference was not statistically significant. Although there were no differences in the maximum GC dose, the current GC dose was significantly higher in the vertebral fracture group than in the nonvertebral fracture group. In addition, a significantly higher proportion of patients in the nonvertebral fracture group were prescribed concomitant active vitamin D3 than those in the vertebral fracture group. Although the lumbar and femoral neck BMD measurements did not differ between the two groups, the GIO scores were significantly higher in the vertebral fracture group than in the nonvertebral fracture group.

Multiple logistic regression analysis identified current GC dose, concomitant active vitamin D3 use, and GIO scores, as factors predicting future vertebral fractures (Table 4). Interestingly, the risk of developing vertebral fractures was 19 times higher in patients receiving bisphosphonate monotherapy than in those receiving bisphosphonates with concomitant active vitamin D3. Unfortunately, due to the small sample size, multiple logistic regression analysis could not be performed for prior fragility fractures.

\section{Discussion}

GCs are commonly used to treat many autoimmune diseases. Although, long-term GC use is associated with various side effects, we found that 97 out of 152 patients (63.8\%) with autoimmune diseases, excluding RA, continued to receive GC treatment in our clinic. The side effects of GC are diverse and can affect the whole body; among them, GIO and secondary vertebral fractures are the most severe adverse events that significantly affect a patient's quality of life [2]. Therefore, in this study, we investigated the treatment of osteoporosis in GC-treated patients with autoimmune diseases. We omitted RA from our analysis because bone destruction and systemic osteoporosis are characteristic of RA [29] and could, therefore, confound our analyses.

Kirigaya et al. [6] investigated the compliance rate to the JSBMR 2004 guidelines based on the health insurance claims database and found that only $23.3 \%$ of the 2,368 patients indicated for osteoporosis treatments were prescribed the recommended medication. Based on this information, the JSBMR updated its guidelines in 2014 to include multiple independent risk factors for GIO to make the guidelines more adaptable in the clinical setting [7]. Our study is the first to evaluate compliance with the JSBMR 2014 guidelines. We found that $63 \%-66 \%$ of patients in our study scored 3 or higher and were indicated for osteoporosis treatment. Of these patients, 93\% received osteoporosis treatments and $50 \%$ received bisphosphonate treatment, demonstrating a much higher compliance rate compared to the JSBMR 2004 guidelines [6]. Interestingly, only two patients did not receive osteoporosis treatment for unknown reasons. This suggests that the updated JSBMR 2014 guidelines are more easily incorporated into clinical practice than the JSBMR 2004 guidelines. However, the compliance rates for the JSBMR 2004 guidelines differed across various departments, with departments of surgery and otolaryngology exhibiting lower adherence compared to the department of internal medicine [6]. Our report is based on the analysis of a single department in a single medical institution. Further studies are needed to investigate the JSBMR 2014 guideline compliance rates across all the clinical departments.

Although there have been reports of ONJ in patients with osteoporosis receiving antiresorptive treatments, the incidence is low, with rates below $0.001 \%$ for bisphosphonatetreated patients [17] and $0.08 \%$ for denosumab-treated patients [30]. Surprisingly, in our study, one (3.3\%) patient taking bisphosphonate and one (8.3\%) patient taking denosumab developed ONJ, which is a relatively high incidence rate compared to other studies of antiresorptivetreated patients. Some studies found that GC use was a risk factor for ONJ [31, 32], possibly because of its immunosuppressant effects, which include slow wound healing and changes in the oral microflora, thereby increasing the risk of infection. However, GC use was not associated with $\mathrm{ONJ}$ in patients with RA [33]. Although we observed a small sample size of patients, our data suggest that it is necessary to analyze the risk of ONJ during GC use in non-RA autoimmune diseases.

During the study period, eight patients developed new vertebral fractures, six of whom received bisphosphonates. Compared to the bisphosphonate-treated patients who did not develop new vertebral fractures, those who developed vertebral fractures had a significantly higher current GC dose, which is consistent with the findings of other studies [34]. This suggests that patients taking a higher dose of GC are at a higher risk of vertebral fractures; however, we did not 
TABLE 3: Characteristics of patients who developed new vertebral fractures during the study period.

\begin{tabular}{|c|c|c|c|c|c|c|c|c|}
\hline Patient & 1 & 2 & 3 & 4 & 5 & 6 & 7 & 8 \\
\hline Age at fracture, (years) & 34 & 49 & 73 & 68 & 75 & 52 & 65 & 44 \\
\hline Underlying disease & SLE & MCTD, HPS & $\mathrm{PM}$ & ILD & PMR & SLE, APS & SLE & TAK \\
\hline Age at onset (years) & 13 & 40 & 72 & 64 & 72 & 23 & 35 & 22 \\
\hline Disease duration (years) & 21 & 9 & 1 & 4 & 3 & 29 & 30 & 22 \\
\hline Max. glucocorticoid dose (mg) & 30 & 90 & 65 & 30 & 15 & 40 & 15 & 40 \\
\hline Glucocorticoid dose at fracture (mg) & 20 & 10 & 13 & 6 & 5 & 8 & 10 & 13 \\
\hline Osteoporosis treatment & None & $\mathrm{D}_{3}$ & BisP & BisP & BisP & $\mathrm{BisP}+\mathrm{D}_{3}$ & BisP & BisP \\
\hline Osteoporosis treatment duration (months) & NA & 6 & 10 & 19 & 26 & 114 & 42 & 180 \\
\hline \multicolumn{9}{|l|}{ BMD, \% YAM } \\
\hline Lumbar spine & 65 & 93 & 84 & 73 & 86 & 91 & 73 & 99 \\
\hline Femoral neck & 60 & 84 & 83 & 75 & 81 & 87 & 90 & 83 \\
\hline \multicolumn{9}{|l|}{ Glucocorticoid-induced osteoporosis score } \\
\hline Lumbar spine & 8 & 4 & 8 & 7 & 5 & 6 & 10 & 11 \\
\hline Femoral neck & 8 & 4 & 8 & 7 & 5 & 6 & 8 & 11 \\
\hline Prior fragility fracture & No & No & No & No & No & No & No & Yes \\
\hline
\end{tabular}

SLE, systemic lupus erythematosus; MCTD, mixed connective tissue disease; HPS, hemophagocytic syndrome; PM, polymyositis; ILD, interstitial lung disease; PMR, polymyalgia rheumatica; APS, antiphospholipid syndrome; TAK, Takayasu arteritis; BMD, bone mineral density; YAM, young adult mean; $\mathrm{D}_{3}$, active vitamin $\mathrm{D}_{3}$; BisP, bisphosphonate.

TABLE 4: Comparison of patient characteristics in patients who received bisphosphonate treatments with and without new vertebral fractures.

\begin{tabular}{|c|c|c|c|c|c|}
\hline & $\begin{array}{l}\text { Without fracture } \\
\quad(n=24)\end{array}$ & $\begin{array}{l}\text { With fracture } \\
\quad(n=6)\end{array}$ & $\begin{array}{l}p \text { value } \\
(t \text {-test })\end{array}$ & $\begin{array}{c}\text { Unadjusted OR } \\
(95 \% \mathrm{CI})\end{array}$ & $\begin{array}{c}p \text { value } \\
\text { (logistic regression) }\end{array}$ \\
\hline Age, (years) & $56(13.1)$ & $63(12.4)$ & 0.25 & $1.05(0.97-1.14)$ & 0.215 \\
\hline Disease duration (years), mean (SE) & $9.5(7.8)$ & $15(13.4)$ & 0.19 & $1.07(0.97-1.17)$ & 0.189 \\
\hline Max. glucocorticoid dose (mg) & $33.1(17.7)$ & $34(18.8)$ & 0.90 & $1.003(0.95-1.06)$ & 0.895 \\
\hline Current glucocorticoid dose (mg) & $6.3(2.7)$ & $9.3(3.2)$ & 0.02 & $1.45(1.02-2.07)$ & 0.022 \\
\hline Combined vitamin $\mathrm{D}_{3}$, no. (\%) & $19(79.2)$ & $1(16.7)$ & 0.0037 & $19(1.79-201.68)$ & 0.015 \\
\hline Prior fragility fracture, no. (\%) & $0(0)$ & $1(16.7)$ & 0.0419 & NA & NA \\
\hline \multicolumn{6}{|l|}{ BMD, \% YAM } \\
\hline Lumbar spine & $93.0(2.4)$ & $84.3(5.6)$ & 0.10 & $0.93(0.85-1.012)$ & 0.076 \\
\hline Femoral neck & $85.6(13.3)$ & $83.2(5.2)$ & 0.67 & $0.98(0.91-1.06)$ & 0.66 \\
\hline \multicolumn{6}{|c|}{ Glucocorticoid-induced osteoporosis score } \\
\hline Lumbar spine & $4.3(1.8)$ & $7.8(2.3)$ & 0.0006 & $2.15(1.18-3.92)$ & 0.001 \\
\hline Femoral neck & $5.0(2.2)$ & $7.5(2.1)$ & 0.0213 & $1.54(1.01-2.34)$ & 0.027 \\
\hline
\end{tabular}

Data are presented as mean (SD) unless otherwise stated. BMD, bone mineral density; $\mathrm{D}_{3}$, active vitamin $\mathrm{D}_{3}$; YAM, young adult mean.

measure the cumulative GC dose, which is also associated with an increased fracture risk [35].

In addition, a significantly lower proportion of patients with new vertebral fractures received concomitant active vitamin D3 compared to those without new vertebral fractures. Importantly, the fracture risk for patients taking bisphosphonates was 19 times higher than for those who did not receive active vitamin D3. These results are consistent with findings from a three-arm randomized controlled study (EDITOR-J Study), which found that the combined use of alfacalcidol and alendronate significantly increased lumbar BMD and reduced the incidence of fractures in patients with GIO compared to treatment with alfacalcidol or alendronate alone [36]. The mechanism for the efficacy of the combined use of alfacalcidol and alendronate is thought to be that active vitamin D3 ameliorates secondary hyperparathyroidism caused by glucocorticoid-induced inhibition of intestinal $\mathrm{Ca}$ absorption, inhibition of renal tubular calcium reabsorption, and shortage of Ca mobilization following alendronate monotherapy. In addition, the effectiveness of bisphosphonates was positively correlated with serum vitamin D levels [37]. As vitamin D insufficiency is common in postmenopausal women [38], vitamin D supplementation is recommended for overall bone health [39]. Guidelines and recommendations for the prevention and treatment of GIO, developed by the American College of Rheumatology, suggest that all patients taking glucocorticoids maintain a vitamin $\mathrm{D}$ intake of 600 to 800 international units/day through either diet and/or supplements [22]. Guidelines on the prevention and treatment of GIO by the French National Authority for Health recommend that loading and maintenance doses of vitamin $\mathrm{D}$ should be administered to elevate the serum 25$\mathrm{OH}$ vitamin D level above the target of $30 \mathrm{ng} / \mathrm{mL}$ in patients with vitamin D insufficiency or deficiency [40]. In Japan, the JSBMR 2014 guidelines also recommend improving nutrition, including dietary calcium and vitamin D intake, maintaining healthy body weight, ceasing smoking and 
alcohol intake, and exercising, for the management of GIO, which is similar to that recommended for the management of primary osteoporosis. However, there are no approved native vitamin D agents (ergocalciferol and cholecalciferol) listed for use with bisphosphonates, and there is no custom of taking native vitamin D supplements; therefore, approved active vitamin D3 agents (alfacalcidol or eldecalcitol) have been used in combination with bisphosphonates. Together, these data support the use of active vitamin $\mathrm{D} 3$ in combination with bisphosphonates for the treatment of GIO, especially in patients with a high fracture risk. However, in our study, only $50 \%$ of bisphosphonate-treated patients also received active vitamin D3.

The mean BMDs in the vertebral fracture group were $84.3 \%$ and $83.2 \%$ in the lumbar and femoral neck, respectively, indicating a relatively normal bone mass. Furthermore, there was no significant difference in BMD between the vertebral and nonvertebral fracture groups. These findings are consistent with studies demonstrating that fractures can occur with minimal or no loss of BMD in patients with GIO $[3,4]$. Our study provides further evidence that BMD should not be the only indicator of osteoporosis treatment in patients with GIO. In addition, the GIO scores calculated on the basis of the JSBMR 2014 guidelines were significantly higher in the vertebral fracture group than in the nonvertebral fracture group, demonstrating the effectiveness of the scoring system in predicting fracture risk.

This study has some limitations. First, as our study was a single-center analysis, we could not determine whether the compliance rate of the JSBMR 2014 guidelines was superior to that of the JSBMR 2004 guidelines. Second, our sample size was small, which may have resulted in selection bias, and large-scale prospective studies encompassing multiple clinics are needed to confirm our findings. Third, we did not measure serum vitamin D levels in these patients. Finally, treatment agents and autoimmune diseases varied considerably. However, previous studies have demonstrated similar risks of GIO and fractures, in many autoimmune diseases [7].

\section{Conclusions}

This study demonstrated a high compliance rate with Japan's GIO treatment guidelines. Based on our results, it might be recommendable that active vitamin $\mathrm{D}_{3}$ be used to reduce the fracture risk associated with bisphosphonates to treat GIO. Large randomized controlled trials should be carried out to confirm our data.

\section{Data Availability}

The datasets associated with the current study are available from the corresponding author upon reasonable request.

\section{Conflicts of Interest}

The authors declare no conflicts of interest.

\section{Acknowledgments}

Editorial support, in the form of medical writing, assembling tables, and creating high-resolution images based on authors' detailed directions, collating author comments, copyediting, fact-checking, and referencing, was provided by Editage and Cactus Communications. The authors would like to express their deepest gratitude to Dr. Hirofumi Taki for his guidance and cooperation in this study.

\section{References}

[1] R. S. Weinstein, "Glucocorticoid-induced bone disease," New England Journal of Medicine, vol. 365, no. 1, pp. 62-70, 2011.

[2] J. Compston, "Glucocorticoid-induced osteoporosis: an update," Endocrine, vol. 61, no. 1, pp. 7-16, 2018.

[3] J. A. Kanis, H. Johansson, A. Oden et al., "A meta-analysis of prior corticosteroid use and fracture risk," Journal of Bone and Mineral Research, vol. 19, no. 6, pp. 893-899, 2004.

[4] T. P. Van Staa, R. F. Laan, I. P. Barton, S. Cohen, D. M. Reid, and C. Cooper, "Bone density threshold and other predictors of vertebral fracture in patients receiving oral glucocorticoid therapy," Arthritis \& Rheumatism, vol. 48, no. 11, pp. 3224-3229, 2003.

[5] H. Nawata, S. Soen, R. Takayanagi et al., "Guidelines on the management and treatment of glucocorticoid-induced osteoporosis of the Japanese society for bone and mineral Research (2004)," Journal of Bone and Mineral Metabolism, vol. 23, no. 2, pp. 105-109, 2005.

[6] D. Kirigaya, T. Nakayama, T. Ishizaki, S. Ikeda, and T. Satoh, "Management and treatment of osteoporosis in patients receiving long-term glucocorticoid treatment: current status of adherence to clinical guidelines and related factors," Internal Medicine, vol. 50, no. 22, pp. 2793-2800, 2011.

[7] Y. Suzuki, H. Nawata, S. Soen et al., "Guidelines on the management and treatment of glucocorticoid-induced osteoporosis of the Japanese society for bone and mineral Research: 2014 update," Journal of Bone and Mineral Metabolism, vol. 32, no. 4, pp. 337-350, 2014.

[8] K. Hu and J. D. Adachi, "Glucocorticoid induced osteoporosis," Expert Review of Endocrinology and Metabolism, vol. 14, no. 4, pp. 259-266, 2019.

[9] K. G. Saag, R. Emkey, T. J. Schnitzer et al., "Alendronate for the prevention and treatment of glucocorticoid-induced osteoporosis," New England Journal of Medicine, vol. 339, no. 5, pp. 292-299, 1998.

[10] J. D. Adachi, K. G. Saag, P. D. Delmas et al., "Two-year effects of alendronate on bone mineral density and vertebral fracture in patients receiving glucocorticoids: a randomized, doubleblind, placebo-controlled extension trial," Arthritis \& Rheumatism, vol. 44, no. 1, pp. 202-211, 2001.

[11] S. A. Stoch, K. G. Saag, M. Greenwald et al., "Once-weekly oral alendronate $70 \mathrm{mg}$ in patients with glucocorticoid-induced bone loss: a 12-month randomized, placebo-controlled clinical trial," Journal of Rheumatology, vol. 36, no. 8, pp. 1705-1714, 2009.

[12] S. Cohen, R. M. Levy, M. Keller et al., "Risedronate therapy prevents corticosteroid-induced bone loss: a twelve-month, multicenter, randomized, double-blind, placebo-controlled, parallel-group study," Arthritis \& Rheumatism, vol. 42, no. 11, pp. 2309-2318, 1999.

[13] D. M. Reid, R. A. Hughes, R. F. J. M. Laan et al., "Efficacy and safety of daily risedronate in the treatment of corticosteroid- 
induced osteoporosis in men and women: a randomized trial," Journal of Bone and Mineral Research, vol. 15, no. 6, pp. 1006-1013, 2000.

[14] S. Wallach, S. Cohen, D. M. Reid et al., "Effects of risedronate treatment on bone density and vertebral fracture in patients on corticosteroid therapy," Calcified Tissue International, vol. 67 , no. 4, pp. 277-285, 2000.

[15] D. M. Reid, S. Adami, J.-P. Devogelaer, and A. A. Chines, "Risedronate increases bone density and reduces vertebral fracture risk within one year in men on corticosteroid therapy," Calcified Tissue International, vol. 69, no. 4, pp. 242-247, 2001.

[16] D. M. Reid, J.-P. Devogelaer, K. Saag et al., "Zoledronic acid and risedronate in the prevention and treatment of glucocorticoid-induced osteoporosis (HORIZON): a multicentre, double-blind, double-dummy, randomised controlled trial," The Lancet, vol. 373, no. 9671, pp. 1253-1263, 2009.

[17] A. A. Khan, A. Morrison, D. A. Hanley et al., "Diagnosis and management of osteonecrosis of the jaw: a systematic review and international consensus," Journal of Bone and Mineral Research, vol. 30, no. 1, pp. 3-23, 2015.

[18] E. Shane, D. Burr, B. Abrahamsen et al., "Atypical subtrochanteric and diaphyseal femoral fractures: second report of a task force of the American Society for Bone and Mineral Research," Journal of Bone and Mineral Research, vol. 29, no. 1, pp. 1-23, 2014.

[19] J. H. Koh, J. P. Myong, J. Yoo et al., "Predisposing factors associated with atypical femur fracture among postmenopausal Korean women receiving bisphosphonate therapy: 8 years' experience in a single center," Osteoporosis International, vol. 28, no. 11, pp. 3251-3259, 2017.

[20] A. Sokal, E. Elefant, T. Leturcq, D. Beghin, X. Mariette, and R. Seror, "Pregnancy and newborn outcomes after exposure to bisphosphonates: a case-control study," Osteoporosis International, vol. 30, no. 1, pp. 221-229, 2019.

[21] N. Machairiotis, G. Ntali, P. Kouroutou, and L. Michala, "Clinical evidence of the effect of bisphosphonates on pregnancy and the infant," Hormone Molecular Biology and Clinical Investigation, vol. 40, 2019.

[22] L. Buckley, G. Guyatt, H. A. Fink et al., "2017 American College of Rheumatology guideline for the prevention and treatment of glucocorticoid-induced osteoporosis," Arthritis \& Rheumatology, vol. 69, no. 8, pp. 1521-1537, 2017.

[23] K. G. Saag, N. Pannacciulli, P. Geusens et al., "Denosumab versus risedronate in glucocorticoid-induced osteoporosis: final results of a twenty-four-month randomized, doubleblind, double-dummy trial," Arthritis \& Rheumatology, vol. 71, no. 7, pp. 1174-1184, 2019.

[24] D. L. Kendler, C. Roux, C. L. Benhamou et al., "Effects of denosumab on bone mineral density and bone turnover in postmenopausal women transitioning from alendronate therapy," Journal of Bone and Mineral Research, vol. 25, no. 1, pp. 72-81, 2010.

[25] J. S. Finkelstein, J. J. Wyland, H. Lee, and R. M. Neer, "Effects of teriparatide, alendronate, or both in women with postmenopausal osteoporosis," Journal of Clinical Endocrinology \& Metabolism, vol. 95, no. 4, pp. 1838-1845, 2010.

[26] K. G. Saag, E. Shane, S. Boonen et al., "Teriparatide or alendronate in glucocorticoid-induced osteoporosis," New England Journal of Medicine, vol. 357, no. 20, pp. 2028-2039, 2007.

[27] R. N. J. de Nijs, J. W. G. Jacobs, A. Algra, W. F. Lems, and J. W. J. Bijlsma, "Prevention and treatment of glucocorticoidinduced osteoporosis with active vitamin D3 analogues: a review with meta-analysis of randomized controlled trials including organ transplantation studies," Osteoporosis International, vol. 15, no. 8, pp. 589-602, 2004.

[28] S. Mori, S. Soen, S. Soen et al., "Justification criteria for vertebral fractures: year 2012 revision," Journal of Bone and Mineral Metabolism, vol. 31, no. 3, pp. 258-261, 2013.

[29] G. Adami and K. G. Saag, "Osteoporosis pathophysiology, epidemiology, and screening in rheumatoid arthritis," Current Rheumatology Reports, vol. 21, no. 7, p. 34, 2019.

[30] S. Papapoulos, R. Chapurlat, C. Libanati et al., "Five years of denosumab exposure in women with postmenopausal osteoporosis: results from the first two years of the FREEDOM extension," Journal of Bone and Mineral Research, vol. 27, no. 3, pp. 694-701, 2012.

[31] F. Jadu, L. Lee, M. Pharoah, D. Reece, and L. Wang, "A retrospective study assessing the incidence, risk factors and comorbidities of pamidronate-related necrosis of the jaws in multiple myeloma patients," Annals of Oncology, vol. 18, no. 12, pp. 2015-2019, 2007.

[32] F. Saad, J. E. Brown, C. Van Poznak et al., "Incidence, risk factors, and outcomes of osteonecrosis of the jaw: integrated analysis from three blinded active-controlled phase III trials in cancer patients with bone metastases," Annals of Oncology, vol. 23, no. 5, pp. 1341-1347, 2012.

[33] Y. Fujieda and T. Atsumi, "Antiresorptive agents-related osteonecrosis of the jaw (ARONJ) in rheumatoid arthritis," Journal of Bone and Mineral Metabolism, vol. 38, no. 5, pp. 739-741, 2020.

[34] T. P. van Staa, H. G. M. Leufkens, L. Abenhaim, B. Zhang, and C. Cooper, "Oral corticosteroids and fracture risk: relationship to daily and cumulative doses," Rheumatology, vol. 39, no. 12 , pp. 1383-1389, 2000.

[35] M. A. Amiche, S. Abtahi, J. H. M. Driessen et al., "Impact of cumulative exposure to high-dose oral glucocorticoids on fracture risk in Denmark: a population-based case-control study," Archives of Osteoporosis, vol. 13, no. 1, p. 30, 2018.

[36] Y. Tanaka, H. Mori, T. Aoki et al., "Analysis of bone metabolism during early stage and clinical benefits of early intervention with alendronate in patients with systemic rheumatic diseases treated with high-dose glucocorticoid: early DIagnosis and Treatment of OsteopoRosis in Japan (EDITOR-J) study," Journal of Bone and Mineral Metabolism, vol. 34, no. 6, pp. 646-654, 2016.

[37] C. Roux, N. Binkley, N. Binkley et al., "Vitamin D status and bone mineral density changes during alendronate treatment in postmenopausal osteoporosis," Calcified Tissue International, vol. 94, no. 2, pp. 153-157, 2014.

[38] M. F. Holick, N. C. Binkley, H. A. Bischoff-Ferrari et al., "Guidelines for preventing and treating vitamin D deficiency and insufficiency revisited," Journal of Clinical Endocrinology \& Metabolism, vol. 97, no. 4, pp. 1153-1158, 2012.

[39] B. Dawson-Hughes, A. Mithal, J.-P. Bonjour et al., "IOF position statement: vitamin $\mathrm{D}$ recommendations for older adults," Osteoporosis International, vol. 21, no. 7, pp. 1151-1154, 2010.

[40] K. Briot, B. Cortet, C. Roux et al., "2014 update of recommendations on the prevention and treatment of glucocorticoid-induced osteoporosis," Joint Bone Spine, vol. 81, no. 6, pp. 493-501, 2014. 